\title{
Partitioning approach for large wind farms: Active power control for optimizing power reserve
}

\author{
Sara Siniscalchi-Minna, Carlos Ocampo-Martinez, Senior Member, IEEE, Fernando D. Bianchi, \\ Mikel De-Prada-Gil, and Bart De-Schutter, Senior Member, IEEE
}

\begin{abstract}
Nowadays, large wind farms are expected to guarantee stability of the electrical grid contributing with ancillary services, such as frequency support. To this end, wind farm controllers must set the power generation of each turbine to compensate generation and demand imbalances. With the aim of optimizing primary frequency support, this paper proposes a partitioning approach to split large wind farms into several disjoint subsets of turbines according to the wake propagations through the wind farm. The partitioning problem is solved as a mixed-integer multi-objective optimization problem stated to maximize the strength of the coupling among the turbines due to the wake effect. Thus, no additional information sharing related to the wake propagations needs to be considered between the subsets. Different control tasks are assigned to the local controller of each subset, such that the total power generated meets the power demanded by the grid while the power reserve for enhancing primary frequency support is maximized. Finally, as an application of the proposed model, a decentralized wind farm control strategy is designed and compared with a centralized approach.
\end{abstract}

\section{INTRODUCTION}

Nowadays, wind power generation supplies more than $30 \%$ of the European electrical consumptions [1]. The evergrowing replacement of conventional power plants by renewable energy systems (RES) has affected the stability of the electrical grid. Therefore, additional requirements are imposed to wind farms, and other RES, in order to provide ancillary services. For instance, the wind farms should maintain supply demand balance by dynamically adjust their power generation to track the power required by the Transmission System Operators (TSOs). Therefore, wind farms need to be controlled with relatively simple control models that regulate the power output to meet the grid requirements [2]. In order to provide frequency control, large wind farms are often operated in de-loading mode. In this circumstance, the power generation is maintained below the available power to keep an amount of power, known as power

S. Siniscalchi Minna and M. De-Prada-Gil are with Catalonia Institute for Energy Research, IREC, Jardins de le Dones de Negre, s/n Barcelona, Spain. \{ssiniscalchi, mdeprada\}eirec.cat

S. Siniscalchi-Minna and C. Ocampo-Martinez are with the Automatic Control Department, Universitat Politècnica de Catalunya, Institut de Ròbotica i Informàtica Industrial (CSIC-UPC), Llorens i Artigas, 4-6, 08028 Barcelona, Spain. \{ssiniscalchi, cocampo\}eiri.upc.edu

F. D. Bianchi is with Instituto Balseiro and CONICET, Bustillo 9500, S.C. Bariloche 8400, Argentina.fernando.bianchi@ib.edu.ar.

B. De-Schutter is with Delft Center for Systems and Control, Delft University of Technology, The Netherlands b. deschutter@tudelft.nI

This work has received funding from the European Union's Horizon 2020 research and innovation programme under the Marie Skodowska-Curie grant agreement No 675318 (INCITE) reserve, which can be used for primary frequency support [3]. During de-loading operation, the power contributions from the turbines within the farm can be regulated for achieving different objectives. For instance, the power dispatch can be chosen for providing inertial control [4], primary [5] or secondary frequency control [6].

For addressing the problem of tracking the power demanded by the TSO while properly regulating the active power generation among the turbines, the most common approach is to use a centralized control strategy. The central controller, according to the information received from the wind turbines, optimizes the control actions, commonly stated as the power set-points or the induction factors, to be sent to each turbine [7]. However, in large wind farms the high amount of information sharing between the turbines and the central controller could be reduced by using decentralized [8] or distributed [9]-[11] control topologies. A consensusbased control for the purpose of maximizing the kinetic energy is proposed in [10], where the authors, to simplify the control design, divide the wind farm into two groups according to the incoming wind speed. Similarly, in [12] the turbines of a large wind farm are split into two groups, one group operating in de-loading mode and the other to maximize the power generation. However, those approaches can cause some loss of power if the distribution of the power set-points among the turbines is not properly optimized. In [11] a distributed strategy is proposed to minimize the turbine interactions by dividing the wind farm in subsets. However, those subsets are defined without considering the strength between the turbines due to the wake effects.

In this paper, a partitioning approach for large wind farms is proposed with the aim of maximizing power reserve whereas ensuring power tracking as well as reducing computational burden, communication issues and complexity of the control design. A mixed-integer multi-objective optimization problem is stated to find the optimal partitions such that the turbines coupled by the wake effect are included into the same partitions. The coupling among turbines is evaluated using a simplified geometrical formulation assuming that the wake expands in a cone-like fashion behind the rotor turbine [13]. Furthermore, a decentralized hierarchical wind farm control strategy is proposed to ensure the tracking of the power demanded by the TSO and to regulate the power set-points for each turbine in case of de-loading operation. The dispatching of the overall power demand is optimized to minimize the wake deficits behind the turbines, i.e., to maximize power reserve that the wind farm can deliver for 
contributing to primary frequency control.

To test the proposed control approach, a wind farm with 30 turbines is considered and the simulations are performed by using SimWindFarm as good-accuracy simulator [14].

The remainder of this paper is organized as follows. The main power formulations and the considered wake effect model are introduced in Section II. The partitioning-related optimization problem is presented in Section III, while the decentralized wind farm controller is stated in Section IV. The main results are shown in Section V. Finally, in Section VI conclusions are drawn.

\section{WIND FARM MODEL}

The power generated by a wind turbine is obtained extracting energy from the incoming wind $v_{i}$ and it is defined as

$$
P_{\mathrm{g}, i}=\kappa_{1} C_{\mathrm{p}}\left(a_{i}\right) v_{i}^{3}=\min \left(P_{\mathrm{av}, i}\left(v_{i}\right), P_{\mathrm{r}, i}\right),
$$

where $\kappa_{1}=\left(\pi \rho R^{2} / 2\right), \rho$ is the air density, $R$ is the rotor radius, $C_{\mathrm{p}}$ is the power coefficient depending on the induction factor $a_{i}, P_{\mathrm{r}, i}$ denotes the power set-point required by the controller, and $P_{\mathrm{av}, i}$ indicates the maximum available power. The latter power is defined as

$$
P_{\mathrm{av}, i}=\min \left(\kappa_{1} C_{\mathrm{p}, \max } \nu_{i}^{3}, P_{\text {rated }}\right),
$$

being $P_{\text {rated }}$ the maximum power capacity of the turbines and $C_{\mathrm{p}, \max }$ the maximum value of the power coefficient obtained for $a_{i}=1 / 3$. In case of high wind speed conditions, some wind turbines are commonly de-loaded, i.e., the power reference $P_{\mathrm{r}, i}$ is set below $P_{\mathrm{av}, i}$. In this case, the difference between available power and the power generated is called power reserve, which is denoted as $P_{\text {res }}$. Notice that, in case of de-loading operation $P_{\mathrm{r}, i}=P_{\mathrm{g}, \mathrm{i}}$, the power generation of each turbine may be determined to ensure specific objectives. For instance, it may be set to minimize the wake effect due to the interaction between the rotor turbine and the incoming wind [15].

Typically, the wind farm layout is chosen to minimize the costs of the electrical inter-connections and to mitigate the wake effects as well as the mechanical loads experienced by the turbines. However, in large wind farms the wake effects are still responsible for a significant amount of power loss, and thus it is desirable to minimize them in order to maximize the wind farm power generation. A possible strategy to achieve this goal with a low computational time consists in dividing the wind farm in subsets according to the wake propagation through the wind farm. For this purpose, a wake model is required. Among the multiple wake models proposed so far [16], suitable estimations of the wake effect on the wind speed crossing the wind farm can be obtained with relatively simple models such as the Jensen's model [13]. Here, the wind speed deficit is modeled as a function of the geographical positions of the wind turbines, the atmospheric wind conditions, and the operating conditions, which affect the induction factor index $a_{i}$. Assuming that the wake expands in a cone-like fashion having circular cross section $2 r_{i}\left(x_{i j}\right)$ for a given free-stream $v_{\infty}$, the downstream wind speed $v_{i}$ at the distance $x_{i j}$ is computed as

$$
v_{i}\left(x_{i j}\right)=v_{\infty}\left(1-2 \sqrt{\sum_{i, j \in \mathbb{N}}\left(2\left(1-a_{j}\right) \frac{r_{0}}{r_{i}\left(x_{i j}\right)}\right)^{2}} \frac{A_{\mathrm{s}, \mathrm{i}}(\phi)}{A_{0}}\right),
$$

where $r_{i}\left(x_{i j}\right)=r_{0}+\alpha\left(x_{j}-x_{i}\right)$ is the radius of the wake generated by the turbine $i$ and $\alpha$ the roughness coefficient, $A_{0}$ and $A_{\mathrm{s}, \mathrm{i}}(\phi)$ are respectively the rotor swap area and the shadowed area of the usptream turbine, which depends to free-stream dominant wind speed direction $\phi$.

Therefore, according to the free-stream wind direction, the wakes generated by the upstream turbines only affect a certain number of downstream turbines. According to this, it is possible to divide large wind farms in a specific number of partitions such that the turbines coupled by the same wake effect may be sorted in the same sub-set. Notice that the strength of the coupling among the turbines due to the wake propagation can be estimated using the following trigonometric relationship [16]:

$$
\varepsilon_{i j}(\phi)=\left|\frac{r_{0}}{r_{i}\left(x_{i j}\right)}\right| \frac{A_{\mathrm{s}, \mathrm{i}}(\phi)}{A_{0}},
$$

where $i, j \in \mathbb{N}_{\geq 1}$ and $i \neq j$. In Section III, it is presented a novel optimization problem to divide the wind farm into several partitions according to the strength $\varepsilon_{i j}$.

\section{iII. Partitioning Problem}

Let the wind farm be considered as a graph $G=(V, E)$ with a set of $n \in \mathbb{Z}_{>0}$ vertices $V=\left\{v_{1}, v_{2}, \ldots, v_{n}\right\}$, corresponding to the turbines within the farm, and a set of edges $E=\{(i, j): i, j \in V\}$ denoting the connections among the turbines (i.e., the couplings due to the wake effect). Furthermore, let $K=\{1,2, \ldots, m\}$ be the set of indices for the $m \in \mathbb{Z}_{\geq 0}$ partitions of the graph $G$ and $\left\{n_{1}, \ldots, n_{l}\right\}$ be the number of turbines within each sub-set. Given the partitioning $P=\left\{V^{l}: l \in K\right\}$, consider the variable $\delta_{i l} \in$ $\{0,1\}$ such that $\delta_{i l}=1$ if $i$ belongs to the sub-set $l$, i.e., $\delta_{i l}=\left\{l \in K: i \in V^{l}\right\}$, and 0 otherwise.

The $m$-partitioning problem is stated to find the optimal set of partitions denoted by $P^{*}$ such that the following objectives are ensured:

Obj1: Minimize the edges between different partitions. This objective may be ensured maximizing the couplings among the turbines due to the wake propagations. Therefore, it can be expressed as the maximization of a function $J_{1}$ that depends on $\varepsilon_{i j}$, i.e.,

$$
J_{1} \triangleq \sum_{l \in K} \sum_{i \in V} \sum_{j \in V \backslash\{i\}}\left(\varepsilon_{i j}+\varepsilon_{j i}\right) \delta_{i l} \delta_{j l}
$$

Obj2: Minimize the distance among the turbines belonging to the same partition, denoted by

$$
J_{2} \triangleq \sum_{l \in K} \sum_{i \in V} \sum_{j \in V \backslash\{i\}} d_{i j} \delta_{i l}
$$

Notice that the latter objective is added to ensure unique solution when no wakes affect the downstream turbines, for instance when the turbines are located in a single row (or column). 
Setting the vector of weigths as $\mathbf{w} \in \mathbb{R}_{>0}^{2}$, the aforemetioned objectives are hierarchically prioritized to find the optimal partition $P^{*}$ by solving the following mixed-integer multi-objective optimization problem:

$$
\begin{array}{ll}
\min _{\delta_{i l}} & \sum_{m=1}^{2} w_{m} J_{m}\left(\delta_{i l}\right), \\
\text { s.t. } & \sum_{i \in V} \delta_{i l} \geq 1, \\
& \sum_{l \in K} \delta_{i l}=1 .
\end{array}
$$

The constraint (7b) is an exclusive constraint, which ensures that every wind turbine can only belong to exactly one subset of $V$. Meanwhile, the constraint (7c) avoids empty partitions. The cost function (7a) is nonlinear but it can be linearized replacing the decision variable with the auxiliary Boolean variable $\delta_{\mathrm{ij} 1} \triangleq \delta_{\mathrm{i} 1} \delta_{\mathrm{j} 1}$ and $\delta_{\mathrm{ij} 1}=1 \leftrightarrow$ $\left(\delta_{\mathrm{il}}=1, \delta_{\mathrm{jl}}=1\right)$. Therefore, the objective function is rewritten as $\sum_{m=1}^{2} w_{m} J_{m}\left(\delta_{\mathrm{ijl}}\right)$ while three additional constraints (i.e., $-\delta_{\mathrm{il}}+\delta_{\mathrm{ijl}} \leq 0,-\delta_{\mathrm{jl}}+\delta_{\mathrm{ijl}} \leq 0$ and $\left.\delta_{\mathrm{il}}+\delta_{\mathrm{jl}}-\delta_{\mathrm{ijl}} \leq 1\right)$ are added at the optimization problem in (7) as proposed in [17].

With the aim of reducing the couplings among different sub-sets, the highest emphasis is put in the first objective. Notice that this objective depends on the wind-flow conditions, hence the optimal problem must be solved every time the wind sped direction changes. Since the computational burden could be quite high, the partioning-related problem can be solved off-line and the optimal partitions updated every time the wind-direction changes.

\section{Partitioning number}

In order to provide the proper number of partitions $m$, this paper proposes a procedure to set the partitions according to the farm layout and dominant free-stream speed direction. In general, the set of turbines $V$ can be split into three sub-sets $V=\left\{V_{\text {up, } v_{\infty}} \cap V_{\text {up }, v_{\mathrm{i}}} \cap V_{\text {down }}\right\}$, where the former set $V_{\text {up, } v_{\infty}}$ contains all the up-stream turbines facing the freestream wind speed $v_{\infty}$, while the latter set $V_{\text {down }}$ includes the most down-stream turbines that are no sources of wake for other turbines. Finally, $V_{\mathrm{up}, \mathrm{v}_{\mathrm{i}}}$ refers to the remaining turbines both receiving and providing wake effects. Moreover, let introduce the wake affecting index $\tau_{i} \triangleq \sum_{j \in V \backslash\{i\}} \varepsilon_{i j}$ that gives information about the amount of wakes that affect the wind speed $v_{i}$ faced by the turbine $i$. Therefore, $\tau_{i}=0$ if $i \in V_{\mathrm{up}, v_{\infty}}$ while the maximum $\tau_{i}$ is obtained for the down-stream turbines most affected by the wakes, i.e., $\tau^{*}=\max _{i \in V_{\text {down }}} \tau_{i}$. Furthermore, the minimum $\tau_{i}$ is identified for the downstream turbines belonging to $V_{\text {down }}$ but affected by only one wake, that is $\tau^{1}=\min _{i \in V_{\mathrm{down}, v_{\mathrm{i}}}} \tau_{i}$. The number of partitions can be chosen such that the aforementioned turbines are clustered with the upstream turbines, since they are not sources of wake for other turbines and are poorly affected by the wakes. On the basis of this idea, let introduce the corelation index $\alpha_{i}=\tau_{i}\left(\tau^{*}\right)^{-1} \in[0,1]$, the number of partitions is determined as the number of turbines $i \in V_{\mathrm{up}, \mathrm{v}_{\mathrm{i}}}$ for which the corresponding parameter satisfies $\alpha_{i} \leq \alpha^{1}$, where $\alpha^{1}=$ $\tau^{1}\left(\tau^{*}\right)^{-1} \in[0,1]$ indicates the minimum co-relation index.

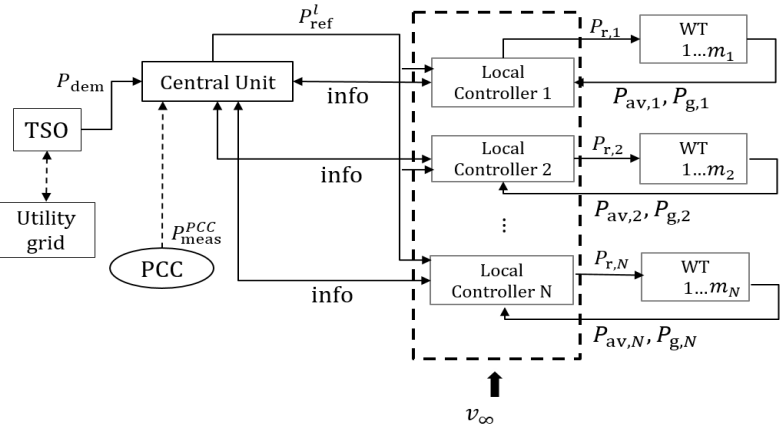

Fig. 1: Wind farm hierarchical control scheme.

\section{Decentralized Control Scheme for Wind FARMS ACTIVE POWER REGULATION}

The hierarchical wind farm decentralized control scheme is shown in Figure 1. The central unit receives the power demand profile $P_{\mathrm{dem}}$ required by the TSO and, according to the information exchange with the local controllers (i.e., available power in the partition $P_{\mathrm{av}}^{l}$ and power generated $P_{\mathrm{g}}^{l}$ for $l \in K)$, computes the power references to be addressed to the local controllers $P_{\text {ref }}^{l}$. Hence, in order to generate the desired power, each controller sets the power set-points for the turbines within the partition $P_{\mathrm{r}, \mathrm{i}}, i \in V^{l}$ considering the available and generated powers of every single turbine. In this work, a de-loading active power control strategy is proposed to guarantee two objectives: 1) dynamically tracking the power demand profile required by the TSO, 2) regulating the power references for each partition and the power set-points for each turbine such that the power reserve of the wind farm is improved.

\section{A. Central Unit Power Dispatch}

In order to reduce the power losses due to the wake effects, an heuristic power dispatch approach to distribute the power demanded by the grid among the $m$-partitions may be focused on prioritizing the power generation of those partitions with low number of down-stream turbines. Meanwhile, the power contribution of those partitions more affected by the wakes should be set to ensure de-loading operation. Consider the set of partition indices to be sorted such that $S=\{l: 1<l<$ $m$ \}, where $l=1$ corresponds to the partition with the lowest number of down-stream turbines and $l=m$ with the partition most affected by the wake propagations. In order to ensure the aforemetioned power dispatch approach, the central unit should solve a linear programming problem stated as

$$
\begin{array}{cl}
\underset{P_{\text {ref }}^{l}, \alpha}{\min } & \varepsilon^{T} \alpha \\
\text { s.t. } & P_{\mathrm{dem}}=\sum_{l=1}^{m} P_{\mathrm{ref}}^{l}, \\
& \left|P_{\mathrm{dem}}-\sum_{k=l}^{m} P_{\mathrm{ref}}^{k}\right| \leq \alpha_{l}, \quad l \in S, \\
& \left|P_{\text {ref }}^{l}-P_{\text {ref }}^{l-1}\right| \leq \alpha_{l}, \quad l>1, l \in S, \\
& P_{\text {min }}^{l} \leq P_{\mathrm{ref}}^{l} \leq P_{\mathrm{av}}^{l}, \quad l \in S,
\end{array}
$$


where $\alpha=\left[\alpha_{1}, \ldots, \alpha_{m}\right]^{T} \in \mathbb{R}^{m}, \varepsilon=\left[\varepsilon_{1}, \ldots, \varepsilon_{m}\right]^{T} \in \mathbb{R}^{m}$ with $\left(\varepsilon_{1}<\varepsilon_{2}<\cdots<\varepsilon_{m}\right)$ are weights. The latter is obtained as the sum over the turbines inside the partition of the wake effects in (4), i.e., $\varepsilon_{l}=\sum_{i, j} \varepsilon_{i, j}$ for $i, j \in V^{l}$ and $l \in S$.

The constraint $(8 \mathrm{~b})$ is added to meet the overall power demand, while the constraint $(8 \mathrm{c})$ guarantees that the maximum and the minimum powers references are respectively required to the first and last partitions. Furthermore, an additional constraint (8d) is added to limit the difference between the power references of two consecutive partitions. Finally, in (8e) the power reference is constrained to be between a maximum and minimum power, given respectively as the sum of the available power and the minimum power for each turbine inside the partition.

\section{B. Predictive Control: Local Controller}

The main objectives of the local controller are

Obj1: Ensure the power reference sent by the central unit.

Obj2: Distribute the power set-points among the turbines in order to maximize the available power (i.e. the power reserve) of each partition.

In order to satisfy the aforementioned objectives, a model predictive control (MPC) strategy is implemented for each local controller. Consider the wind turbine system to be controlled with a dynamical model given by

$$
\mathbf{x}_{l, k+1}=\mathbf{A}_{l, d} \mathbf{x}_{l, k}+\mathbf{B}_{l, d} \mathbf{u}_{l, k}+\mathbf{B}_{l, d_{1}} \mathbf{d}_{l, k}
$$

where $k \in \mathbb{Z}_{\geq 0}$ denotes the discrete-time step, $\mathbf{x}_{k}^{l}=$ $\left[\mathbf{P}_{\mathrm{g}, k}^{T}, \xi\right]^{T} \in \mathbb{R}^{n_{x}^{l}}$ where $n_{x}^{l}=n^{l}+1$ with $n^{l}$ denoting the number of turbines at each partition and $\mathbf{u}_{l, k}=\mathbf{P}_{\mathrm{r}, k}^{T} \in \mathbb{R}^{n_{u}^{l}}$ are respectively the vector of system states and manipulated variables. Furthermore, $\mathbf{P}_{\mathrm{g}, k} \in \mathbb{R}^{n_{l}}$ and $\mathbf{P}_{\mathrm{r}, k} \in \mathbb{R}^{n_{l}}$ indicate the vector of generated and required powers for the turbines in the partition $l$, and $\xi$ is an integral action to ensure a zero steady-state tracking error. Moreover, let $\mathbf{d}_{l, k}=P_{\text {ref }}^{l}$ be the power reference. Finally, the matrices $\mathbf{A}_{l, d}, \mathbf{B}_{l, d}$ and $\mathbf{B}_{l, d_{1}}$ are the discrete-time version of the continous-time matrices

$$
\mathbf{A}_{l}=\left[\begin{array}{cc}
-\frac{1}{\tau} \mathbf{I}_{n_{x}^{l}} & 0 \\
-\mathbf{1}_{n_{x}^{l}} & 0
\end{array}\right], \quad \begin{aligned}
\mathbf{B}_{l} & =[1 / \tau, \ldots, 1 / \tau, 0]^{T}, \\
\mathbf{B}_{l, 1} & =[0, \ldots, 0,1]^{T},
\end{aligned}
$$

depending on the time constant used to model the wind turbine as a first-order system. Notice that the validity of this model has been already shown in a previous work of the authors [5].

In order to evaluate the optimal sequence of the control action $\mathbf{u}_{k}^{l *}=\left[P_{\mathrm{r}, 1}^{*}, \ldots, P_{\mathrm{r}, \mathrm{n}_{1}}^{*}\right]$ to be addressed at the turbines, the following multi-objective optimization problem should be solved:

$$
\begin{array}{ll}
\min _{\hat{\mathbf{u}}_{l}} & \sum_{j=1}^{m} \beta_{j} f_{j}\left(\mathbf{x}_{l, k}, \hat{\mathbf{u}}_{l, k}\right) \\
\text { s.t. } & \mathbf{x}_{l,(k+j+1 \mid k)}=\mathbf{A}_{d} \mathbf{x}_{l,(k+j \mid k)}+\mathbf{B}_{l, d} \mathbf{u}_{l,(k+j \mid k)}+\mathbf{B}_{l, d 1} \mathbf{d}_{l,(k+j \mid k)}, \\
& \mathbf{u}_{l,(k+j \mid k)} \in \mathbb{U},
\end{array}
$$

for $j \in\left[0, H_{p}-1\right]$ with $H_{p}$ the prediction horizon and $\mathbb{U}=$ $\left\{\mathrm{P}_{\mathrm{r}} \in \mathbb{R}^{n_{u}} \mid P_{\mathrm{r}}(k) \in\left[\underline{P}_{\mathrm{r}}, \bar{P}_{\mathrm{r}}\right]\right\}$. Notice that the multi-objective cost function in (11) is defined to solve the objectives Obj1 and Obj2. Specifically, the tracking is obtained as the minimization of the objective function $f_{1}=\left(Q \mathbf{x}_{k, l}\right)^{\prime}\left(Q \mathbf{x}_{k, l}\right)$ with $Q=[0, \ldots, 0,1] \in \mathbf{R}^{n_{x}}$. Notice that the previous objective is equal to $\left|P_{\mathrm{ref}}^{l}-P_{\mathrm{g}, t o t}^{l}\right|$, where $P_{\mathrm{g}, t o t}^{l}$ denotes the total power generated in the partition $l$. The second objective, that is the maximization of power reserve, can be achieved by setting the power reference $P_{\mathrm{r}, i}$ in order to reduce the wind speed deficits behind the turbines, i.e., to improve the incoming wind for the downstream turbines. Since there exists a oneto-one relationship between the induction factor $a_{i}$ and $P_{\mathrm{r}, i}$, the latter can be chosen such that the incoming wind for the downstream turbines in (3) is improved. A possible approach to ensure this goal is to require more contribution to the total generated power for the most down-stream wind turbines while minimizing the power contribution of the most upstream turbines. As presented in [18], this second objective can be stated using a weighted sum of the power generated, i.e., $f_{2}=\sum_{i: i \in V^{l}} \gamma_{i} x_{k, i}$. Here, the indices $i$ are sorted according to the free-stream wind speed and the wind farm layout in order to have $\left\{i: 1 \leq i \leq n^{l}\right.$ for $v_{i} \geq v_{j}$ with $i<j, i, j \in$ $\left.V^{l}\right\}$. The index $i=1$ corresponds to the turbine less affected by the wake and $i=n^{l}$ to the turbines facing the highest number of wakes, hence the weighthing elements $\gamma_{i}$ should be set to ensure $\gamma_{1} \geq \gamma_{2} \geq \ldots \gamma_{n}$.

Finally, an additional objective is included to smooth the variation of the manipulated variables and to avoid peaks on the power set-points, objective that is denoted as $f_{3}=$ $\left(R \Delta \mathbf{u}_{k, l}\right)^{\prime}\left(R \Delta \mathbf{u}_{k, l}\right)$, with $\Delta \mathbf{u}_{k, l}=\mathbf{u}_{k, l}-\mathbf{u}_{k-1, l}$ and $R=\mathbf{I}_{n^{l}}$.

\section{Case Study}

The proposed control strategy was evaluated for a wind farm with 30 benchmark NREL-5MW wind turbines spaced $5 D$ (i.e. $630 \mathrm{~m})$ in $(\mathrm{x}, \mathrm{y})$-directions and placed in a $(5 \times 6)$ matrix, see Figure 2. The wind field and wake effect have been simulated with SimWindFarm [14], a Simulink toolbox for wind farm simulation and control. The free-stream wind speed has been set equal to $11 \mathrm{~m} / \mathrm{s}$ with a prevailing wind direction of 30 degrees.

To show the effectiveness of the proposed wind farm control strategy, two case studies are discussed. First case: the partitioning optimization problem together with the definition of the partitioning number are tested for both the wind farm and wind conditions under consideration. Second case: the proposed decentralized control performance in terms of tracking of the power demand profile and power reserve maximization is shown. In order to highlight the benefits achieved using the proposed approach, decentralized control strategy (DMPC) has been compared with the centralized predictive control (C-MPC) approach as reported in [18].

\section{A. Partitioning Results}

In order to solve the proposed mixed-integer optimization problem, the weighting vector in (7a) has been set to $\mathbf{w}=[1,0.3]^{T}$. Four optimal partitions were found applying 


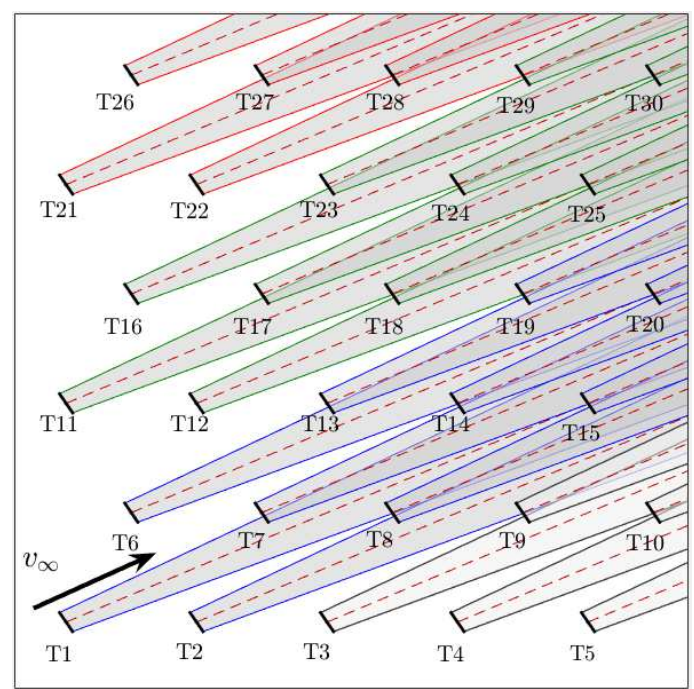

Fig. 2: Optimal phisical partition for a wind speed direction of 30 degrees.

the partitioning procedure presented in Section III. The minimum and maximum wake affecting indices have been found equal to $\tau^{l}=0.181$ and $\tau^{*}=0.346$, corresponding respectively to the wake affecting index of the turbines T27 and T30. Therefore, the number of partitions has been computed as the number of downstream turbines having the wake co-relation index $\alpha_{i}$ lower or equal than the minimum value $\alpha_{l}=0.523$, i.e., the turbines T9-T10-T27-T28.

As stated in Section III, the aforementioned turbines belong to the partitions containing only the upstream turbines, i.e., the first (grey contour line) and second partitions (red contour line). Meanwhile, those turbines more affected by the wakes are equally included into the third (blue contour line) and fourth partitions (green contour line). The benefits of this partitioning approach in terms of maximization of the power reserve are shown next.

\section{B. Closed-Loop Performance}

The time constant $\tau$ used for modeling turbines as a first order systems has been set to $0.08 \mathrm{~s}$, the MPC strategy has been implemented for a sampling time $T_{s}=0.01 \mathrm{~s}$ and the prediction horizon has been set to $H_{p}=4$. The prioritization of the objective functions in (11) was ensured by setting the weighting vector $\beta=[10,5,1]^{T}$. To evaluate the power reference tracking of the wind farm controller, Figure 3 shows the system response for a power demand profile increasing from $80 \mathrm{MW}$ until $120 \mathrm{MW}$. It can be observed that the power generated $P_{\mathrm{g}}$ (grey dashed line) tracks the power demand $P_{\mathrm{dem}}$ (red line) until $t_{2}=500 \mathrm{~s}$. For $t>t_{2}$, the power generated follows the available power $P_{\mathrm{av}}$ (blue line); in fact, in this condition the tracking cannot be ensured being the available power $P_{\mathrm{av}}$ lower than the power demand.

The power references $P_{\text {ref }}^{j}$ required by the central unit for each partition are shown in Figure 4. According to the power dispatch strategy in (8), the power references for the first and

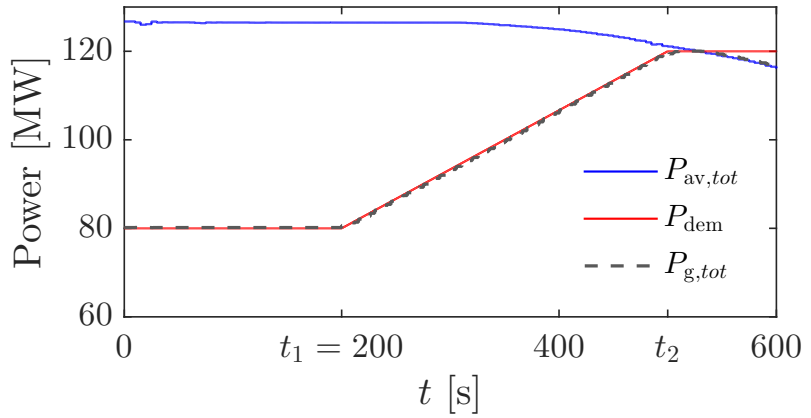

Fig. 3: Closed-loop performance for power reference tracking at wind farm control level. Power generated $P_{\mathrm{g}}$, power demand $P_{\text {dem }}$ and available power $P_{\mathrm{av}}$.
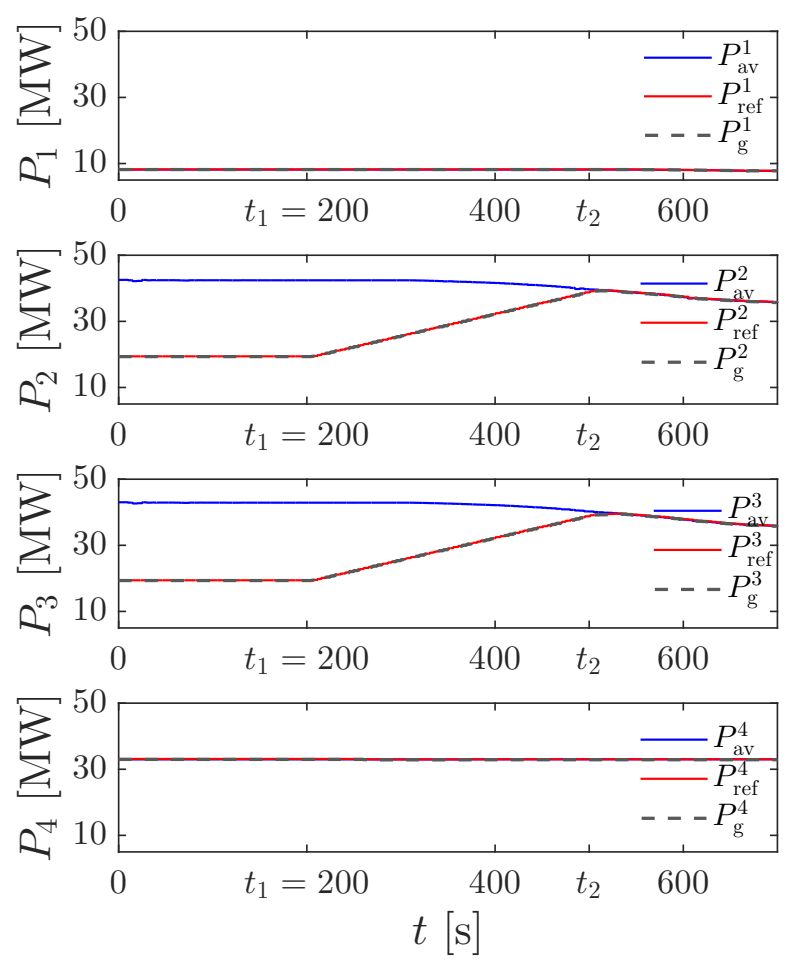

Fig. 4: System response for power reference tracking at central unit control level.

second partitions (red line) are set equal to the maximum available power (blue line), such that $P_{\text {ref }}^{1}=9.8 \mathrm{MW}$ and $P_{\text {ref }}^{2}=32 \mathrm{MW}$. For the other partitions highly affected by the wake propagations, the optimal power references are distributed to ensure de-loading operation. Specifically, $P_{\text {ref }}^{3}$ and $P_{\text {ref }}^{4}$ increase from $14 \mathrm{MW}$ at $t \leq 200 \mathrm{~s}$ until reaching the available power at $t=t_{2}$.

In order to ensure the power references desired from the central unit, the first and second local controllers set the power generation $P_{\mathrm{g}, i}$ of each turbine to meet the available power $P_{\mathrm{av}, i}$. Meanwhile, for the partitions working in deloading mode, different contributions are required for each 
TABLE I: Percentage values of $\left(P_{\mathrm{g}, \mathrm{i}} / P_{\mathrm{av}, \mathrm{i}}\right) \%$. Partition 1.

\begin{tabular}{|c|c|c|}
\hline \multicolumn{2}{|c|}{$\left(P_{\mathrm{g}, \mathrm{i}} / P_{\mathrm{av}, \mathrm{i}}\right) \%$ : Partition 1} \\
\hline \hline Parition 1 & T3 & T9 \\
\hline DMPC & 100 & 100 \\
\hline CMPC & 21,92 & 100 \\
\hline
\end{tabular}

TABLE II: Percentage values of $\left(P_{\mathrm{g}, \mathrm{i}} / P_{\mathrm{av}, \mathrm{i}}\right) \%$. Partition 4.

\begin{tabular}{|c|c|c|c|c|c|c|c|}
\hline \multicolumn{2}{|c|}{$\left(P_{\mathrm{g}, \mathrm{i}} / P_{\mathrm{av}, \mathrm{i}}\right) \%$ : Partition 4 } & & & & \\
\hline \hline Turbine & T11 & T12 & T17 & T18 & T24 & T25 & T30 \\
\hline DMPC & 28,67 & 29,17 & 99,45 & 99,46 & 99,80 & 99,91 & 99,98 \\
\hline CMPC & 66.15 & 66.15 & 66.15 & 100 & 100 & 100 & 100 \\
\hline
\end{tabular}

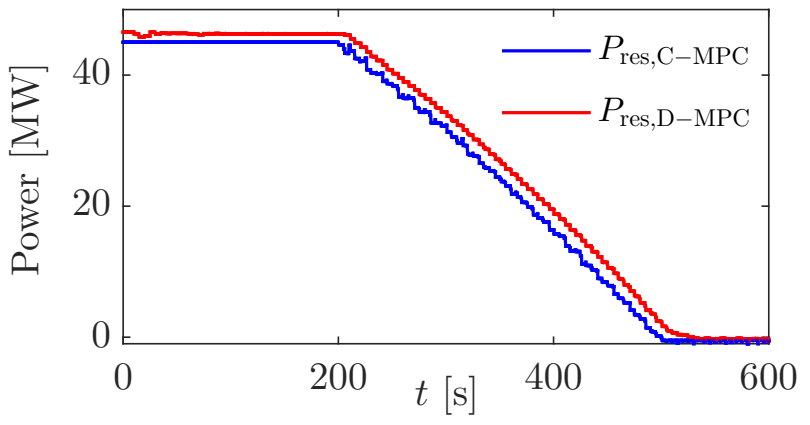

Fig. 5: Power reserve of the wind farm for decentralized (DMPC) and centralized (CMPC) approach.

turbine. According to the active power regulation proposed by adding the objective $f_{2}$ in (11), the power contribution of the most downstream turbines is maximized while it is minimized for the upstream turbines. In Table I and Table II, the values of power generated $P_{\mathrm{g}, \mathrm{i}}$ for the turbines in the first partition and for some turbines in the fourth partition are listed. Such values are given as percentage of the available power $P_{\mathrm{av}, \mathrm{i}}$ for a constant value of $P_{\mathrm{dem}}=100 \mathrm{MW}$. Furthermore, for comparison purposes, power generation values are listed also for the CMPC approach. Neglecting the wake effect on the downstream turbines in the partitions less affected by the wakes the DMPC requires the maximum power to those turbines. Therefore, the power generated by the turbines in the partitions more affected by the wakes is reduced more than the case solved with CMPC and this decreases the wind speed deficits behind those turbines. This fact results in increasing power reserve of the overall wind farm. Figure 5 shows the power reserve profiles for DMPC $P_{d e m, D M P C}$ (red line) and for CMPC $P_{d e m, C M P C}$ (blue line). The mean value of power reserve obtained with the proposed approach results to be increased about $11,5 \%$ with respect to the centralized strategy. Therefore, the performance of the proposed control strategy in providing power reserve for enhancing primary frequency support is improved.

\section{CONCLUSIONS}

This paper has proposed a partitioning approach to divide large wind farms into several disjoint partitions in order to ease the massive information exchange between the turbines and the wind farm central controller. The number of partitions and the assignment of turbines to the resultant partitions have been found in function of the wake propagation through the wind farm. A decentralized control approach has been proposed to gurantee both the tracking of the power demanded by the TSO and the maximization of the power reserve. Specifically, the latter goal has been achieved by cleverly distributing the power reference among the partitions such that the overall wake effect is minimized. The effectiveness of the proposed approach has been verified by comparing the decentralized approach with a centralized strategy, where the results have shown that the power reserve has been increased of $11,5 \%$ with respect to the centralized control scheme.

\section{REFERENCES}

[1] WindEurope. (2017, Sep.) Wind energy in europe, scenarios for 2030. [Online]. Available: https://windeurope.org/about-wind/reports

[2] S. Boersma, B. Doekemeijer, P. Gebraad, P. Fleming, J. Annoni, A. Scholbrock, J. Frederik, and J. van Wingerden, "A tutorial on control-oriented modeling and control of wind farms," in American Control Conference (ACC), 2017, 2017, pp. 1-18.

[3] Y.-Q. Bao and Y. Li, "On deloading control strategies of wind generators for system frequency regulation," International Transactions on Electrical Energy Systems, vol. 25, no. 4, pp. 623-635, 2015.

[4] A. De Paola, D. Angeli, and G. Strbac, "Scheduling of wind farms for optimal frequency response and energy recovery," IEEE Transactions on Control Systems Technology, vol. 24, no. 5, pp. 1764-1778, 2016.

[5] S. Siniscalchi-Minna, F. Bianchi, and C. Ocampo-Martinez, "Predictve control of wind farms based on lexicographic minimizers for power reserve maximization," in Proc. of American Control Conference $(A C C)$. IEEE, 2018.

[6] C. Shapiro, P. Bauweraerts, J. Meyers, C. Meneveau, and D. F. Gayme, "Model-based receding horizon control of wind farms for secondary frequency regulation," Wind Energy, vol. 20, pp. 1261-1275.

[7] P. A. Fleming, "Active power control of waked wind farms: Preprint," National Renewable Energy Lab.(NREL), Golden, CO (United States), Tech. Rep., 2017.

[8] S. Asadollah, R. Zhu, M. Liserre, and C. Vournas, "Decentralized reactive power and voltage control of wind farms with type-4 generators," in PowerTech, 2017 IEEE Manchester. IEEE, 2017, pp. 1-6.

[9] S. Baros and M. D. Ilić, "Distributed torque control of deloaded wind DFIGs for wind farm power output regulation," IEEE Transactions on Power Systems, vol. 32, no. 6, pp. 4590-4599, 2017.

[10] X. Gao, K. Meng, Z. Y. Dong, D. Wang, M. S. El Moursi, and K. P. Wong, "Cooperation-driven distributed control scheme for large-scale wind farm active power regulation," IEEE Transactions on Energy Conversion, vol. 32, no. 3, pp. 1240-1250, 2017.

[11] J. Annoni, C. Bay, T. Taylor, L. Pao, P. Fleming, and K. Johnson, "Efficient optimization of large wind farms for real-time control," in 2018 Annual American Control Conference (ACC), June 2018, pp. 6200-6205.

[12] W. Zhang, Y. Xu, W. Liu, F. Ferrese, and L. Liu, "Fully distributed coordination of multiple DFIGs in a microgrid for load sharing," IEEE Transactions on Smart Grid, vol. 4, no. 2, pp. 806-815, 2013.

[13] N. O. Jensen, "A note on wind generator interaction," Roskilde, Denmark, Tech. Rep., 1983.

[14] J. D. Grunnet, M. Soltani, T. Knudsen, M. N. Kragelund, and T. Bak, "Aeolus toolbox for dynamics wind farm model, simulation and control," in The European Wind Energy Conference \& Exhibition, EWEC, 2010.

[15] S. Siniscalchi-Minna, F. D. Bianchi, M. De-Prada-Gil, and C. OcampoMartinez, "A wind farm control strategy for power reserve maximization," Renewable Energy, vol. 131, pp. 37-44, 2019.

[16] R. J. Barthelmie, K. Hansen, S. T. Frandsen, O. Rathmann, and Schepers, "Modelling and measuring flow and wind turbine wakes in large wind farms offshore," Wind Energy, vol. 12, no. 5, pp. 431-444, 2009

[17] A. Bemporad and M. Morari, "Control of systems integrating logic, dynamics, and constraints," Automatica, vol. 35, no. 3, pp. 407-427, 1999.

[18] S. Siniscalchi-Minna, M. De Prada Gil, F. Bianchi, C. OcampoMartinez, and B. De Schutter, "A multi-objective predictive control strategy for enhancing primary frequency support," in Journal of Physics: Conference Series, 2018. 